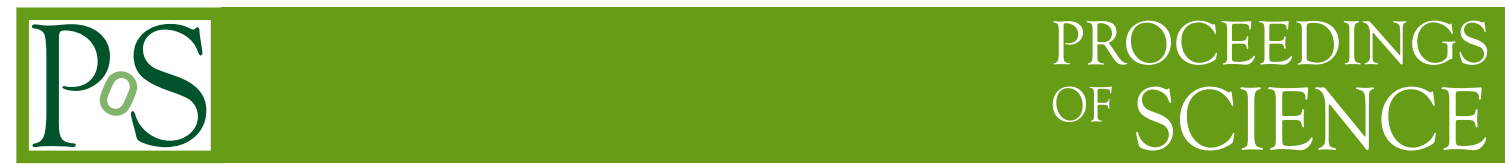

\title{
X(3872) Transport at the LHC
}

\section{Biaogang Wu, ${ }^{a, *}$ Xiaojian Du, ${ }^{b}$ Matthew Sibila ${ }^{c}$ and Ralf Rapp ${ }^{a}$}

${ }^{a}$ Cyclotron Institute and Department of Physics and Astronomy, Texas A\&M University,

College Station, TX 77843-3366, USA

${ }^{b}$ Fakultät fur Physik, Universität Bielefeld,

D-33615 Bielefeld, Germany

${ }^{c}$ Department of Physics \& Astronomy, Ohio Northern University,

Ada, OH 45810, USA

E-mail: bgwu@tamu.edu, xjdu@physik.uni-bielefeld.de, m-sibila@onu.edu, rapp@comp.tamu.edu

The production yields of the $X(3872)$ particle in heavy-ion collisions have been suggested to be sensitive to its internal structure. While the thermal equilibrium limit of the $X(3872)$ abundance only depends on its mass, its inelastic reaction rates in a hot medium are expected to be different depending on whether it is a loosely bound hadronic molecule or a compact tetraquark dominated by colored diquark-antidiquark configurations. Here we implement these scenarios into a kinetic rate equation as previously used in applications to charmonium and bottomonium observables. While the equilibrium limit, controlled by the charm-quark content in the fireball, is universal for both scenarios (and decreases with temperature), the molecular scenario is characterized by a much larger width in the hadronic medium than for the tetraquark. Consequently, the average production time of the molecular $X(3872)$ is significantly later than for the tetraquark, leading to to a factor of $\sim 2$ smaller production of the former compared to the latter. This outcome is qualitatively different from most coalescence model calculations to date. In addition, we calculate pertinent transverse-momentum spectra, which turn out to be harder for the molecular scenario due to the larger blue-shift caused by the collective flow in the fireball at later times.

HardProbes 2020

1-6 June 2020

Austin, Texas

${ }^{*}$ Speaker 


\section{Introduction}

The constituent-quark content of the $X$ (3872)particle remains an open question to date. The leading candidates are a compact tetraquark structure with a diquark of charm and light quark $(c q)$ and its anti-diquark vs. a loosely bound hadronic molecule structure of a $\mathrm{D}$ and $D *$ meson [1]. It has been suggested the production systematics of the X(3872)in ultra-relativistic heavy-ion collisions (URHICs) can provide additional insights into this question, as the different structures should lead to rather different reaction rates and melting temperatures in the hot fireball, especially in the hadronic phase of its evolution. Specifically, the molecular state is expected to develop much larger reactions rates in hadronic matter than the compact tetraquark, since the former's wave function has a much larger overlap with hadronic final states than the latter's.

In the present work we implement the different scenarios into our transport approach which has been extensively used in the simulations of charmonium and bottomonium states in URHICs at the SPS, RHICH and the LHC [2-4]. In particular, we will compute the centrality and transversemomentum dependent production yields suitable for comparisons to future observables.

\section{Transport Approach in Thermal Medium}

The bulk medium evolution of our transport approach utilizes a time-dependent blast-wave description with typical evolution time scales taken from hydrodynamic simulations. The total entropy at each centrality is adjusted to the experimentally observed hadron abundances, and the thermal freezeout temperature and flow velocities are compatible with empirical extractions from the transverse-momentum $\left(p_{T}\right)$ spectra of various light-hadron species. In the present work we focus on $\mathrm{Pb}-\mathrm{Pb}$ collisions at $5 \mathrm{TeV}$, for which typical temperature evolutions are shown in Fig. (1),

Given the bulk evolution model, we evaluate the chemical reactions of the $X(3872)$ from a rate equation including dissociation and formation channels,

$$
\frac{d N_{X}(\tau)}{d \tau}=-\Gamma(T(\tau))\left[N_{X}(\tau)-N_{X}^{\mathrm{eq}}(T(\tau))\right]
$$

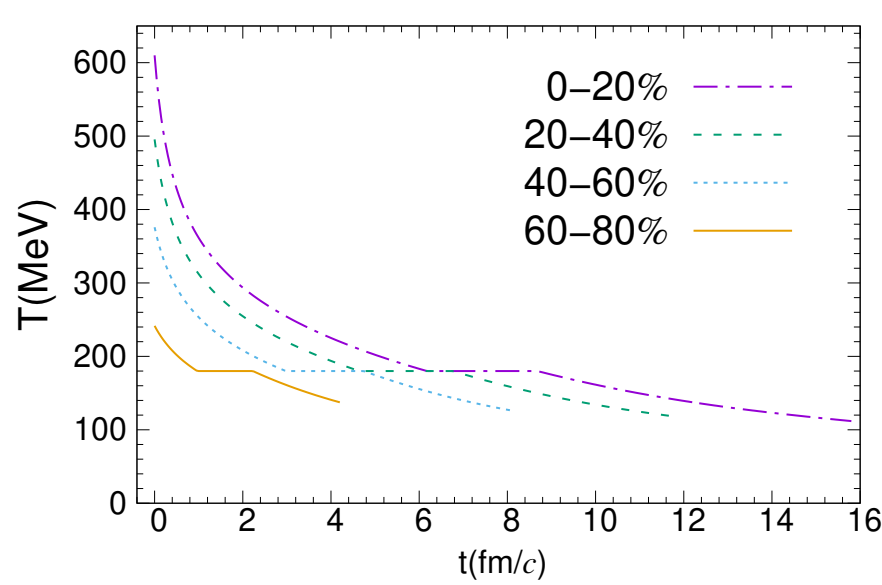

Figure 1: Temperature evolution of thermally expanding fireball in $5 \mathrm{TeV} \mathrm{Pb}-\mathrm{Pb}$ collisions including both parton and hadron stages at different centralities 
The number of $X(3872)$ particles, $N_{X}$, is governed by two transport parameters. The first one is the equilibrium limit,

$$
N_{X}^{\mathrm{eq}}(T(\tau))=d_{X} \gamma_{c}^{2} \int \frac{d^{3} k}{(2 \pi)^{3}} \exp \left(-\sqrt{k^{2}+m_{X}^{2}} / T\right),
$$

with the $X(3872)$ 's degeneracy, $d_{X}=3$, and mass, $m_{X}$. The charm-quark fugacity factor accounts for both charm and the anti-charm quarks within the $X(3872)$, and is calculated from charm conservation in the thermal statistical model,

$$
N_{c \bar{c}}=\frac{1}{2} \gamma_{c} n_{\mathrm{op}} V_{\mathrm{FB}} \frac{I_{1}\left(\gamma_{c} n_{\mathrm{op}} V_{\mathrm{FB}}\right)}{I_{0}\left(\gamma_{c} n_{\mathrm{op}} V_{\mathrm{FB}}\right)}+\gamma_{c}^{2} n_{\mathrm{hid}} V_{\mathrm{FB}},
$$

with $N_{c \bar{c}}$ the total number of charm-anticharm pairs in the fireball at given centrality.

The second transport parameter is the inelastic reaction rate, $\Gamma$, of the $X(3872)$. Instead of employing a specific model calculation, we parameterize the rates based on generic structure arguments with guidance from the existing literature [5-7]. For the loosely bound molecule we assume a range of rather large widths, $\Gamma_{0}^{\mathrm{mol}} \simeq(400 \pm 100) \mathrm{MeV}$ at an initial temperature of $T_{0}=180 \mathrm{MeV}$, while for the compact tetraquark we use $\Gamma_{0}^{\text {tet }}=50-80 \mathrm{MeV}$ at $T_{0}$, about an order of magnitude smaller. This hierarchy, with $\Gamma_{0}^{\mathrm{mol}} \gtrsim T_{0} \geq \Gamma_{0}^{\text {tet }}$, implies that the tetraquark can survive at medium temperatures higher than in the hadronic phase (i.e., in the QGP phase) while the molecule cannot. Thus we define initial conditions for our rate equation where at the beginning of the hadronic phase the $X(3872)$ is at its chemical-equilibrium value for the tetraquark and zero for the molecule scenario. [8] (we neglect primordial production). The temperature dependence of the inelastic reaction rates is simulated with a power law dependence,

$$
\Gamma(T)=\Gamma_{0}\left(\frac{T}{T_{0}}\right)^{n},
$$

, similar to the case of the hadronic reaction rates for charmonia [4]. For definiteness, we here focus on $n=3$, but variations of this exponent have rather little impact on our results [8].

\section{Time Evolution and Observables for $X(3872)$ Production}

The time evolution of the $X(3872)$ yield per unit rapidity following from the solution of our rate equation for the two structure scenarios is displayed in Fig. 2, for central and semi-central $\mathrm{Pb}-\mathrm{Pb}$ collisions at $5.02 \mathrm{TeV}$ at mid-rapidity. We first note that the $X(3872)$ 's equilibrium number in the fireball decreases with time; despite a large increase in the fugacity factor, the thermal penalty of its large mass cannot be overcome. In the tetraquark scenario, the small widths in the hadronic phase lead to a very small reduction of its chemical-equilibrium value inherited from the QGP phase. On the other hand, for the molecular configuration, the $X$ (3872)rather rapidly approaches chemical equilibrium at the later stages, especially for more central collisions and for higher dissociation temperatures (below which regeneration sets in). As a result, the molecule scenario produces significantly smaller yields than the tetraquark. This still holds if the molecule were initialized at the chemical-equilibrium value, due to the large reaction rates driving the yields toward the equilibrium at later stages.

The calculated centrality dependence (normalized to the number of binary nucleon-nucleon collisions to mimic a nuclear modification factor, $R_{\mathrm{AA}}$ ) are displayed in Fig. 3. For both scenarios 

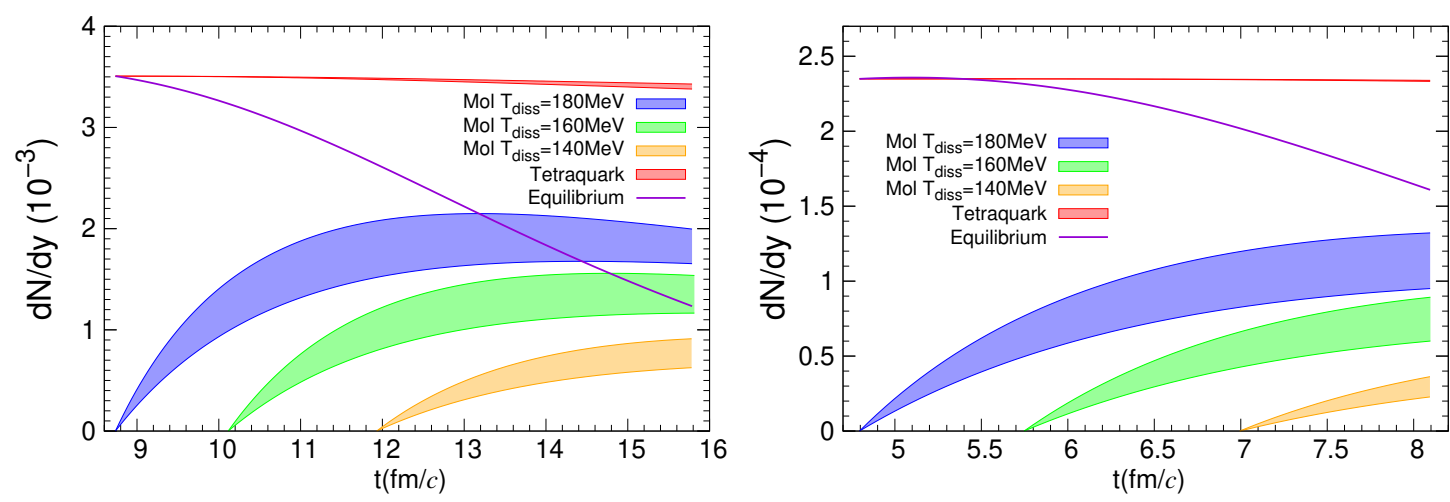

Figure 2: Time evolution of the $X$ (3872)for the molecular (lower bands) and tetraquark (upper red band) scenarios for the hadronic phase in $0-20 \%$ (left panel) and $40-60 \%$ (right panel) $\mathrm{Pb}-\mathrm{Pb}$ collisions at $5 \mathrm{TeV}$. The bands reflect width ranges with $\Gamma_{0}^{\mathrm{mol}}=300-500 \mathrm{MeV}$ and $\Gamma_{0}^{\mathrm{tet}}=50-80 \mathrm{MeV}$; the blue, green and orange bands for the molecular scenario are for different dissociation temperatures of $T_{\text {diss }}=180,160$ and $140 \mathrm{MeV}$, respectively. The purple lines indicate the equilibrium limit.
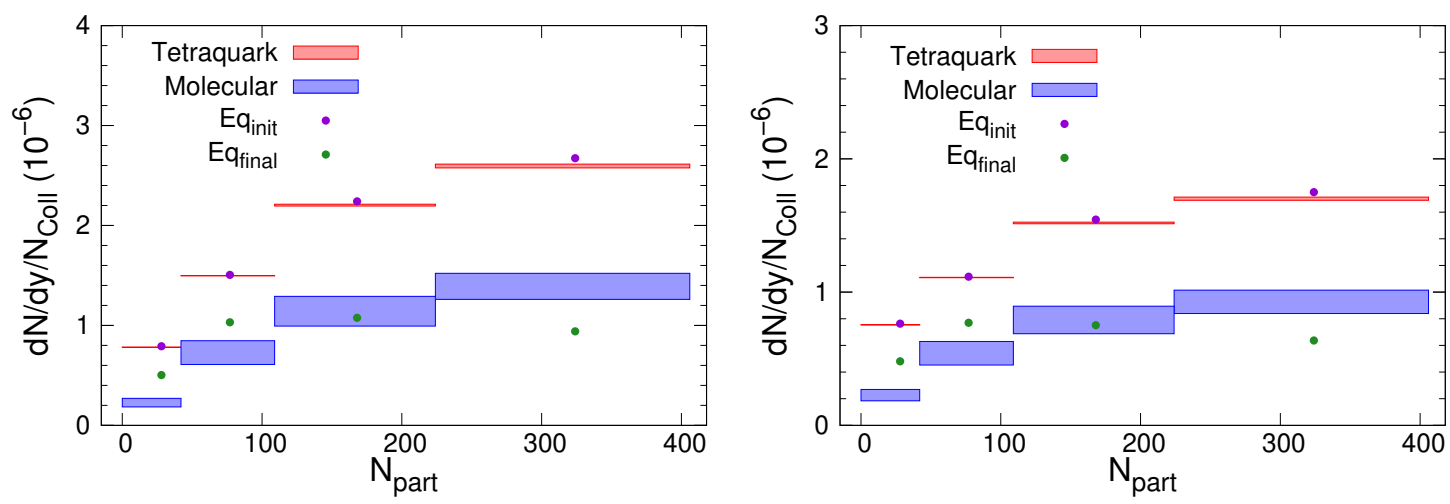

Figure 3: Centrality dependence of the $X(3872)$ production yields with (right) and without (left) shadowing effect, normalized to the number of primordial $N N$ collisions (red bars: tetraquark, blue bars: molecule, with $T_{\text {diss }}=180 \mathrm{MeV}$ ). Also shown are the equilibrium values at chemical and thermal freezeout (symbols).

a rising trend with centrality is found, with a tetraquark over molecule ratio of around 2 (except for the most peripheral bin). Including up to $20 \%$ nuclear shadowing in charm production in central collisions, the $X(3872$ ) yields are suppressed by up to $\simeq 40 \%$, see right panel of Fig. 3 .

The different production times also result in different $p_{T}$ spectra of the $X(3872)$ in the different scenarios, cf. Fig. 4. The earlier formation of tetraquarks leads to softer spectra (close to the blastwave result at $T=180 \mathrm{MeV}$ ), while the molecular spectra are closer to the blastwave spectra at kinetic freezeout $T=110 \mathrm{MeV}$, more so for lower dissociation temperatures.

\section{Conclusions}

We have conducted transport calculations of $X(3872)$ production in URHICs within a welltested rate-equation approach. Different internal structures of the $X(3872)$, characterized by a scale hierarchy of type $\Gamma^{\mathrm{mol}} \gtrsim T_{c} \geq \Gamma^{\text {tet }}$, lead to a sequential formation of $X(3872)$ states in the 


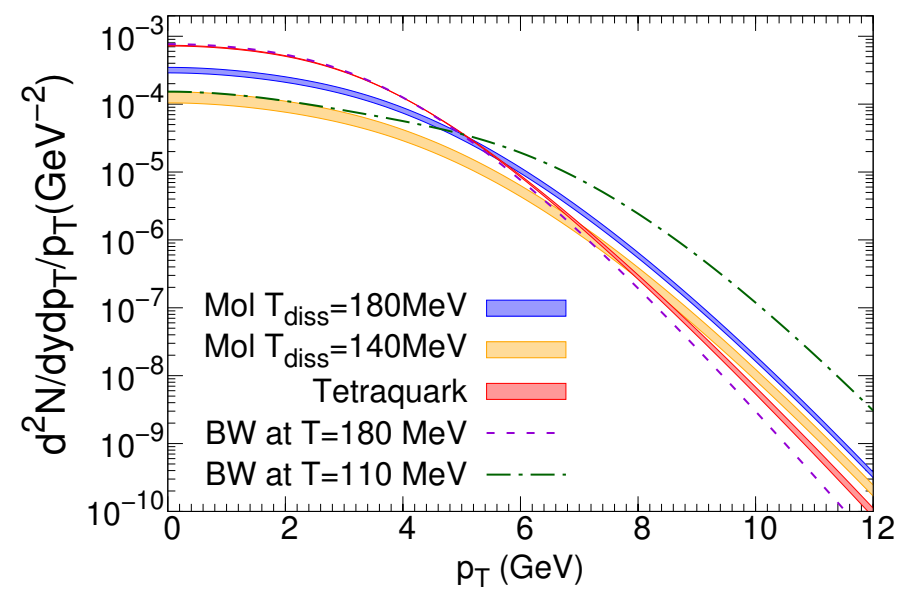

Figure 4: $X(3872) p_{T}$ spectra in $0-20 \%$ central $\mathrm{Pb}-\mathrm{Pb}$ collisions for the molecular (blue (orange) band for $T_{\text {diss }}=180(140) \mathrm{MeV}$ ) and tetraquark (red band) scenarios, compared to blastwave spectra at chemical (dashed line) and thermal (dash-dotted line) freezeout.

two scenarios. The resulting yields of the tetraquark are roughly twice as large (and their $p_{T}$ spectra significantly softer) compared to the molecule, which differs from most coalescence model predictions.

Acknowledgments. This work is supported by the U.S. NSF under grant no. PHY-1913286.

\section{References}

[1] A. Esposito, A. Pilloni and A. Polosa, Multiquark Resonances, Phys. Rept. 668 (2017) 1.

[2] L. Grandchamp, R. Rapp and G.E. Brown, In medium effects on charmonium production in heavy ion collisions, Phys. Rev. Lett. 92 (2004) 212301.

[3] X. Zhao and R. Rapp, Medium Modifications and Production of Charmonia at LHC, Nucl. Phys. A 859 (2011) 114.

[4] X. Du and R. Rapp, Sequential Regeneration of Charmonia in Heavy-Ion Collisions, Nucl. Phys. A 943 (2015) 147.

[5] M. Cleven, V.K. Magas and A. Ramos, X(3872) in a hot pion bath, Phys. Lett. B 799 (2019) 135050.

[6] F. Brazzi, B. Grinstein, F. Piccinini, A.D. Polosa and C. Sabelli, Strong Couplings of $X(3872)_{J=1,2}$ and a New Look at J/ $\psi$ Suppression in Heavy Ion Collisions, Phys. Rev. D 84 (2011) 014003.

[7] E.G. Ferreiro and J.-P. Lansberg, Is bottomonium suppression in proton-nucleus and nucleus-nucleus collisions at LHC energies due to the same effects?, JHEP 10 (2018) 094.

[8] B. Wu, X. Du, M. Sibila and R. Rapp, X(3872) Transport in Heavy-Ion Collisions, 2006.09945. 